\title{
Electron beam relaxation in inhomogeneous plasmas
}

\author{
A. Voshchepynets and V. Krasnoselskikh \\ LPCE/CNRS UMR7328, Orleans, France
}

Correspondence to: A. Voshchepynets (woschep@gmail.com)

Received: 12 March 2013 - Revised: 17 June 2013 - Accepted: 5 July 2013 - Published: 8 August 2013

\begin{abstract}
In this work, we studied the effects of background plasma density fluctuations on the relaxation of electron beams. For the study, we assumed that the level of fluctuations was so high that the majority of Langmuir waves generated as a result of beam-plasma instability were trapped inside density depletions. The system can be considered as a good model for describing beam-plasma interactions in the solar wind. Here we show that due to the effect of wave trapping, beam relaxation slows significantly. As a result, the length of relaxation for the electron beam in such an inhomogeneous plasma is much longer than in a homogeneous plasma. Additionally, for sufficiently narrow beams, the process of relaxation is accompanied by transformation of significant part of the beam kinetic energy to energy of accelerated particles. They form the tail of the distribution and can carry up to $50 \%$ of the initial beam energy flux.
\end{abstract}

Keywords. Space plasma physics (wave-particle interactions; waves and instabilities)

\section{Introduction}

Understanding the generation of type III solar radio bursts is a long-standing problem in space plasma physics (Parker, 1965). Solar type III radio bursts are produced by fast electron beans (energy from several $\mathrm{keV}$ to several tens of $\mathrm{keV}$ ) ejected in the anti-sunward direction during solar flares (Lin, 1970). Radio bursts have a characteristic frequency near the local electron plasma frequency and/or its harmonic. Such a mechanism of generation was proposed by Ginzburg and Zhelezniakov (1958) and further refined by Papadopoulos et al. (1974). It is widely accepted that these radio bursts are generated as a result of the electron beam-plasma interaction. Emission on the fundamental frequency (the plasma frequency) and its harmonic (twice the plasma frequency) is generated by nonlinear processes that occur following the primary generation of Langmuir waves by the electron beam.

The excitation of Langmuir waves in plasmas by electron beams is one of the first kinetic instabilities described by quasi-linear theory (Vedenov et al., 1962; Drummond and Pines, 1962), and studied in controlled laboratory experiments. However, during the beginning of these studies it was determined that the quasi-linear theory of beam relaxation in homogeneous plasma results in a length of relaxation which in solar corona conditions should be approximately several hundred km (Sturrock, 1964), while electron beams, generated in the solar atmosphere, are observed in interplanetary space for the orbits of Earth and Jupiter (Buttighoffer, 1998). During the process of beam relaxation, the region of the positive slope of the electron distribution function responsible for the generation of primary Langmuir waves should shift with time toward lower velocities. On the other hand, inhomogeneous plasma waves can stay in phase with beam particles only for a finite time due to the change of the $\boldsymbol{k}$ vector, and, consequently, the phase velocity. As a result, the efficiency of the wave particle interaction is significantly diminished (i.e. the energy transfer from particles to waves is slowed). The process of formation for the plateau of the velocity distribution instead of the beam is considerably reduced. Another important effect that results in the reduction of the efficiency of the "plateauing" process is due to the re-absorption of Langmuir waves by electrons from the velocity range where the slope is negative (Zaitsev et al., 1972). Numerical simulations (Takakura and Shibahashi, 1976; Grognard, 1982) have demonstrated that the beam can propagate out to a distance of about $1 \mathrm{AU}$, and predict plateau-like electron distributions. However, the electron beams detected at $1 \mathrm{AU}$ and associated with solar type III radio bursts (Lin et al., 1981) do not contain any plateau-type features in the distribution function.

Nonlinear plasma processes, such as induced scattering on ions, development of weak or strong turbulence of Langmuir 
waves (Zakharov, 1972; Shapiro and Shevchenko, 1984) may significantly change the Langmuir wave spectrum, and, therefore, slow down the beam relaxation.

The spectrum of density fluctuations obtained during ISEE inter-satellite propagation experiment (Celnikier et al., 1983) indicates that the solar wind plasma is strongly inhomogeneous. Variations of the density reconstructed from the spectrum (Kellogg, 1986; Kellogg et al., 1999) indicates that inhomogeneities can impact Langmuir wave propagation and can influence the transport of solar flare produced energetic electrons from the Sun to the Earth (Reid and Kontar, 2010; Zaslavsky et al., 2010; Foroutan et al., 2008; Kontar and Reid, 2009; Ziebell et al., 2011; Li et al., 2006). Worth noting is that Ergun et al. (2008) found that some observed Langmuir waves can be trapped within solar wind density cavities.

This leads to the necessity of reformulating the problem of beam plasma interactions by taking into account the effects of plasma density inhomogeneities. Hereafter, we suppose that the effect of Langmuir wave trapping within preformed density cavities takes place everywhere in the system and determines the wave dynamics during beam relaxation. For the work presented here, we studied the evolution of the electron beam velocity distribution function, $\bar{f}$ using equations for the quasi-linear relaxation of electron beams in strongly inhomogeneous plasmas as derived by Ryutov (1969).

\section{Model description}

In this work, we considered the same one-dimensional system as in Ryutov (1969) where the overwhelming majority of waves are assumed to be trapped inside density depletions in the vicinity of the local minima of the plasma density. The profile around the minimum can be approximated by parabola. In order to calculate the averaged over space increment and the diffusion coefficient, Ryutov (1969) initially evaluated these parameters for one single cavity and found that they can be presented in the form of integrals that are dependent on the particle velocity at the bottom of the well and do not depend on the well parameters. This universality allowed him to perform averaging over space. The result was shown to be independent of the details of the cavity distribution and was presented in a universal form that was dependent only on the average wave energy density, $\bar{W}$, and the averaged particle distribution function, $\bar{f}$ (for more details see the Appendix), as follows:

$$
\begin{aligned}
& \frac{\partial \bar{f}}{\partial t}=\frac{\partial}{\partial v}\left[\frac{\partial \bar{f}}{\partial v} \int_{0}^{v} \frac{\bar{W} d u}{u^{2} \sqrt{v^{2}-u^{2}}}\right], \\
& \frac{\partial \bar{W}}{\partial t}=u \bar{W} \int_{u}^{\infty} \frac{\partial \bar{f}}{\partial v} \frac{v d v}{\sqrt{v^{2}-u^{2}}},
\end{aligned}
$$

where $v$ is the electron velocity, $u$ is the phase velocity of Langmuir waves at the bottom of the density cavity (the dependence of $v$ on $u$ is determined using Eq. A16). An analytical solution of the system was obtained by Ryutov (1969) only when the initial dispersion of the beam $\Delta v$ is negligible in comparison with the beam velocity $v_{0}: v_{0} \gg \Delta v$. Here, we present the numerical solution of the system (1, 2). Below, we describe and discuss the dynamics of the beam distribution function, $\bar{f}$, and the averaged wave energy density, $\bar{W}$. To solve the system $(1,2)$, we applied the finite difference method with the second-order, semi-implicit scheme (Samarskii and Popov, 1975). As for the initial conditions for $\bar{f}$ we used the Gaussian distribution with the mean value normalized to the beam velocity $v_{0}\left(v_{0}=1\right.$ arbitrary units) and the variance $\Delta v$ (for e.g. $\Delta v=0.06,0.08,0.1,0.2,0.3$ ). The initial distribution of $\bar{W}$ within the phase space was uniform. As shown in Ryutov (1969), the system has the following invariant:

$\frac{d}{d t}\left[\frac{1}{2} \int_{0}^{\infty} v^{2} \bar{f} d v+\int_{0}^{\infty} \frac{\bar{W}}{u^{3}} d u\right]=0$.

The first term corresponds to the particle kinetic energy $\epsilon_{\mathrm{k}}$, the second - to the wave energy $\epsilon_{\mathrm{w}}$. Initially, the ratio of $\epsilon_{\mathrm{w}}$ to $\epsilon_{\mathrm{k}}$ is chosen to be $\epsilon_{\mathrm{w}} / \epsilon_{\mathrm{k}}=0.0001$.

\section{Results}

The dynamics of $\bar{f}$ obtained in the simulation is shown in Fig. 1. On the right and left panels one can see the snapshots of $\bar{f}$ for different moments of time. In a homogeneous plasma, relaxation stops when within the entire range of velocities the distribution satisfies the condition $\partial \bar{f} / \partial v \leq 0$ (Vedenov et al., 1962), corresponding to the absence of instability. The time of relaxation in dimensionless units, as determined using the details provided in the Appendix (following Eq. A26), is on the order of $\tau \approx 1$. As shown in Fig. 1, at the end of the period the relaxed beam distribution, $\bar{f}$, still has a positive slope. The dynamics of $\bar{W}$ obtained in the simulation is shown in Fig. 2. The maximum value of $\bar{W}$ obtained in simulation was $\bar{W}_{\max }=0,15$ in arbitrary units (as shown in the Appendix, after Eq. A26). We normalized $\bar{W}$ to $\bar{W}_{\text {max }}$ in Fig. 2 . By comparing the dynamics of $\bar{f}$ and the wave energy $\epsilon_{\mathrm{w}}$ one can notice that the process of the positive slope decrease corresponding to conventional formation of the plateau of the electron distribution ("plateauing") continues to operate while $\epsilon_{\mathrm{W}}$ continues to grow (in Figs. 1 and 2 it corresponds to time interval $t=0.0, t=0.5$ ). After this time, $\epsilon_{\mathrm{w}}$ begins to decay but particle diffusion is still at work for some time. For the conditions of simulation as presented in the Fig. 1 the relaxation process ends after $\tau \approx 1$.4. As shown in the Appendix, we obtain an expression describing the averaged growth rate of the wave, $\bar{\gamma}$, dependence on the electron distribution function. 

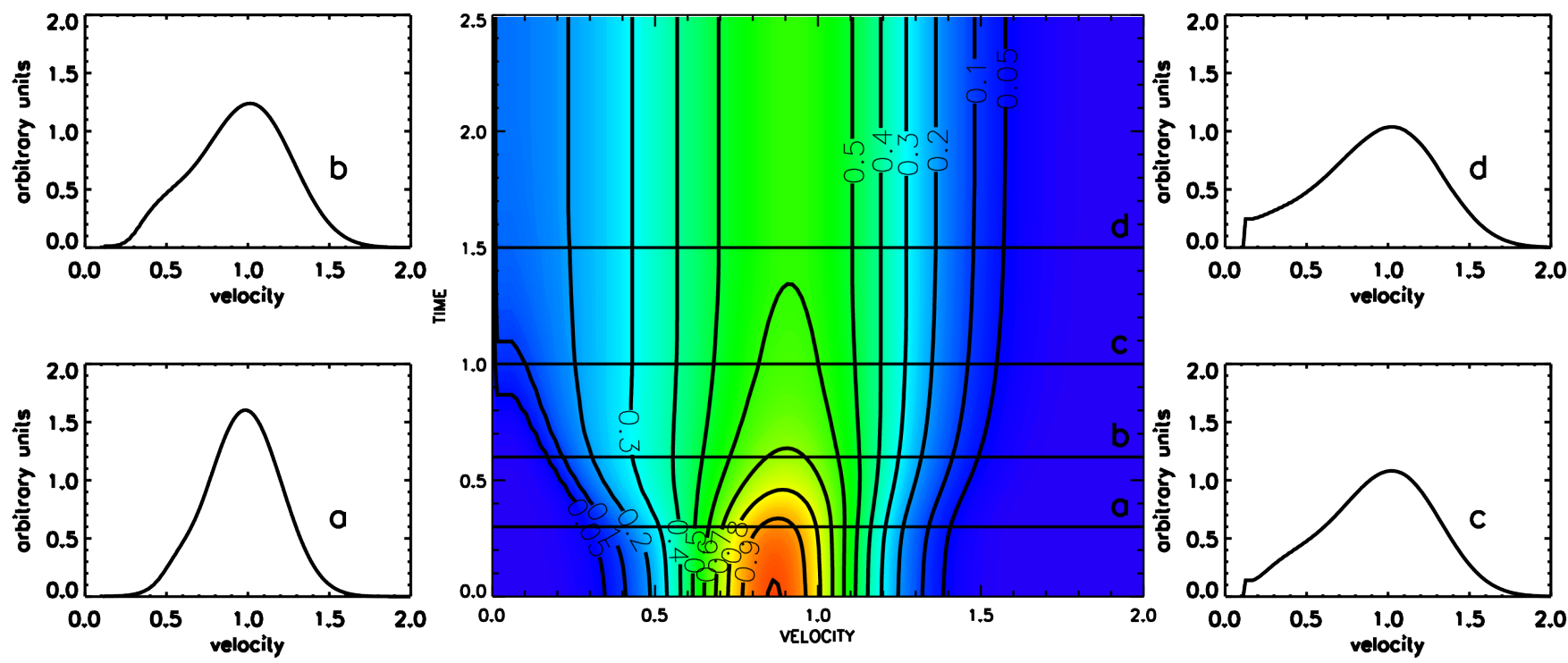

Fig. 1. The dynamics of the electron distribution function obtained from the numerical simulation (shown in the central panel). The small panels provide snapshots of the distribution function. The values of the distribution function and the velocity are provided in arbitrary units (in the Appendix following Eq. A26). The width of the initial Gaussian is $\Delta v=0.2$.
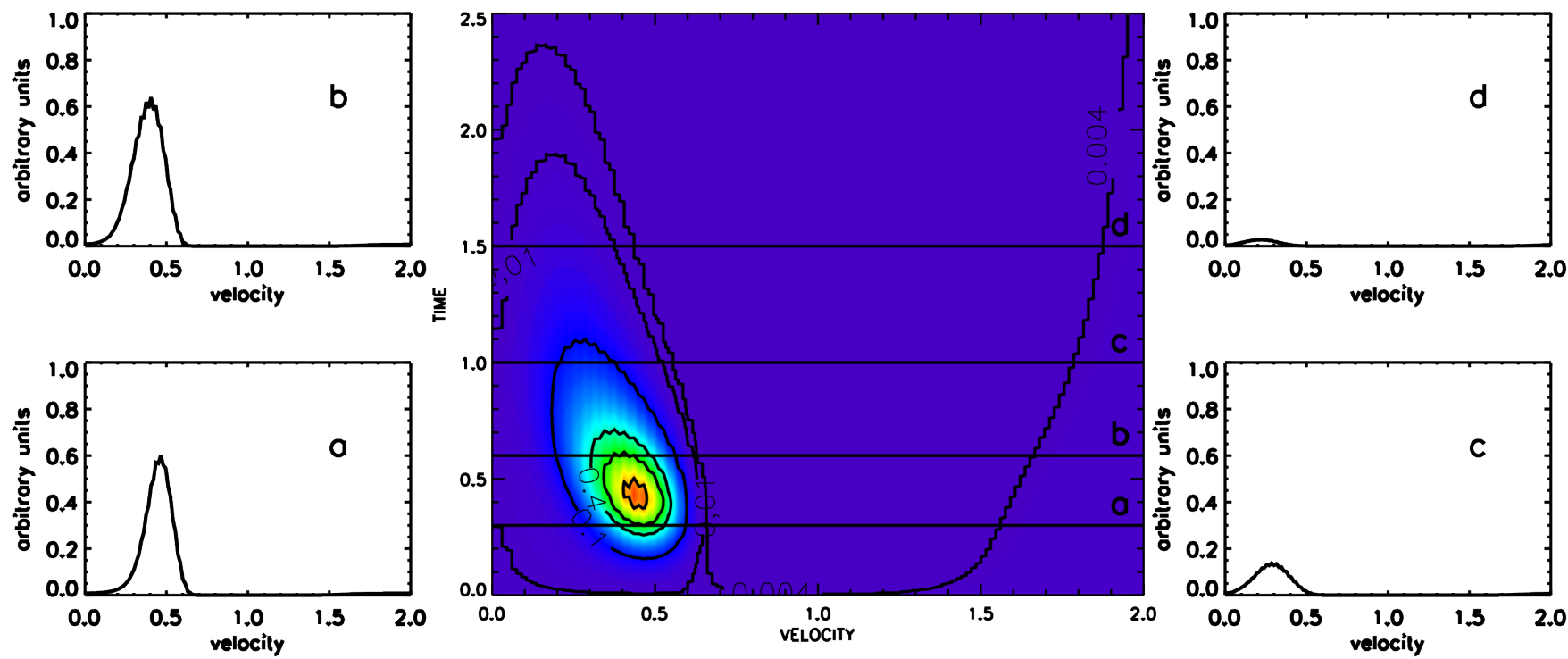

Fig. 2. The dynamics of the averaged wave spectral power obtained using the numerical simulation, as shown in the central panel. Small panels provide snapshots of the wave spectral power. The values of the wave spectral power and the velocity are provided in arbitrary units (in the Appendix following Eq. A26).

In Fig. 3 the dependencies of the distribution function and the growth rate on velocity are presented for two moments in time. As one can see, the impact of wave trapping on inhomogeneous plasma as described by the averaged $\bar{\gamma}$ results in significant reduction of the range of phase velocities $u$ where waves can be generated. Very important difference between beam plasma interaction in homogeneous and inhomogeneous plasmas consists in the direct relation of the slope of distribution with the positiveness of growth rate in homogeneous plasma and absence of such relation in inhomogeneous, indeed the waves can damp in the areas of the velocity space where the distribution function has positive slope. The effect appears due to the variation of the wave's phase velocity in inhomogeneous plasma. The majority of waves that are trapped pass a significantly longer time in areas where they are damped than in areas where they can grow. Therefore, the effect of averaging along the wave's trajectory explains the change in the relationship between the 

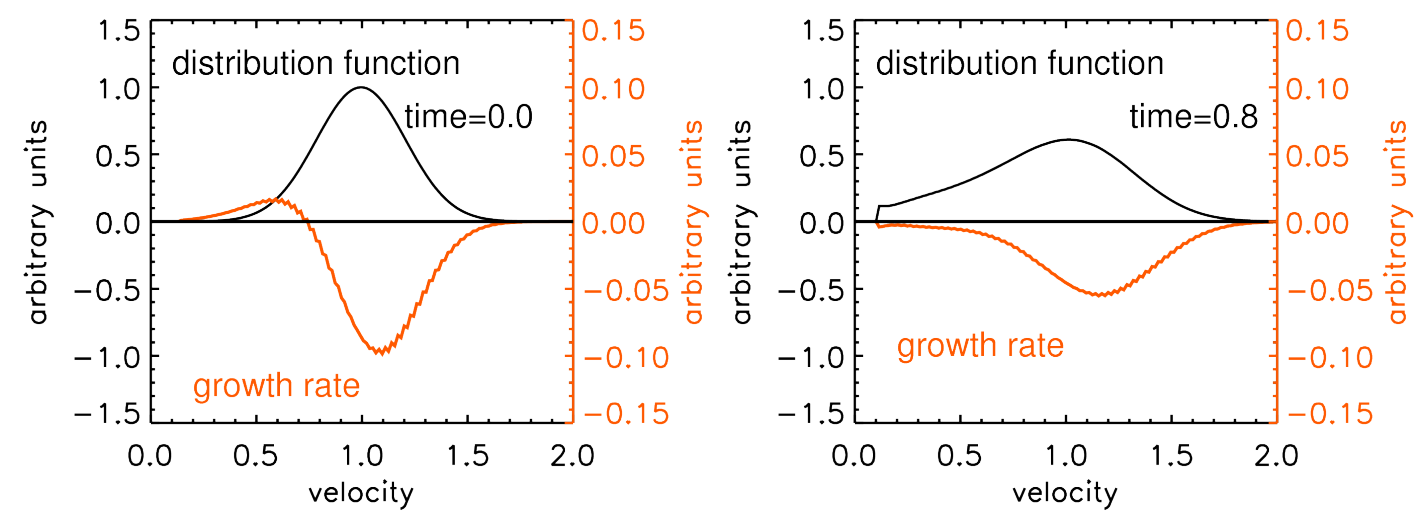

Fig. 3. The electron distribution function $\bar{f}$ (black) and the related Langmuir wave growth rate $\bar{\gamma}$ (red) (the $x$ and $y$ axis are same as in Fig. 1).
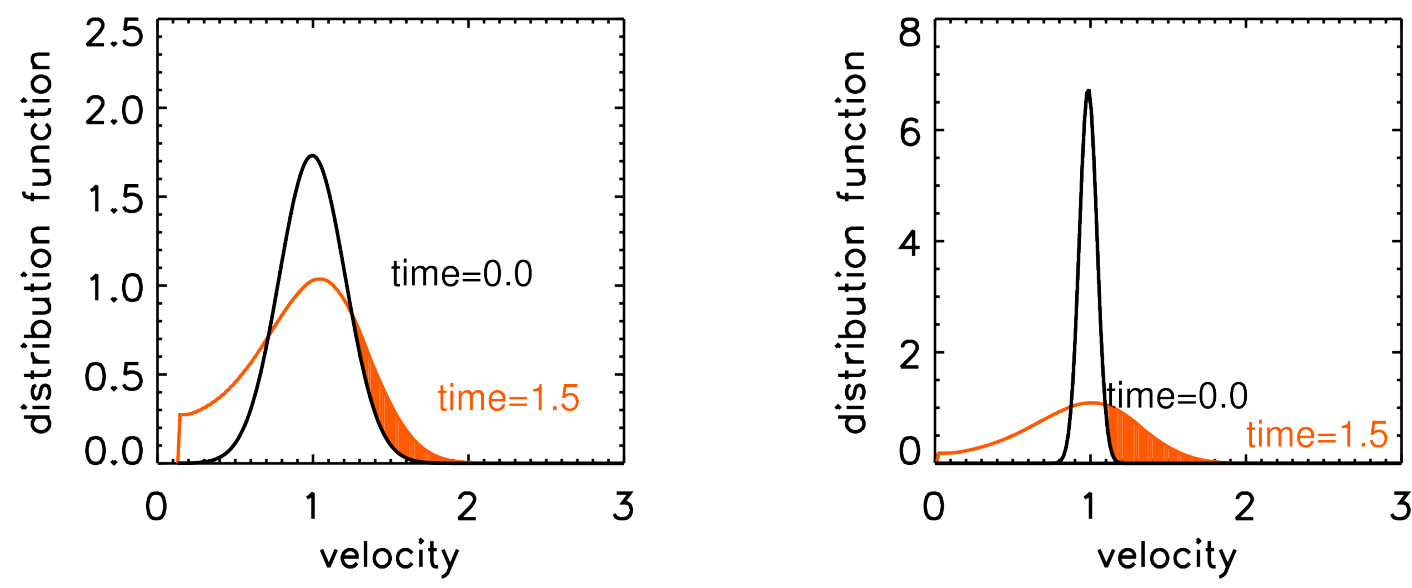

Fig. 4. $\bar{f}$ for different moments of time (in arbitrary units). The initial electron beam distribution, $\bar{f}$, is plotted in black and the final distribution, $\bar{f}$, is plotted in brown. The right and left panels correspond to the different width of the beam with respect to its energy $\Delta v$ $(\Delta v=0.2$ and $\Delta v=0.08)$ within the velocity space.

slope of the velocity distribution and wave growth/damping. The process of plateau formation can take place only if the wave intensity in the corresponding region is strong. Thus, relaxation of the beam significantly slows down due to the shrinking of the region where waves grow $\gamma>0$. The region becomes narrower with time and from the moment shown in the left panel in Fig. 3, we have an $\gamma<0$ for all waves. After $\tau=0.8$ the waves could only be damped and the formation of the plateau eventually stopped, electrons begin to reabsorb the energy of Langmuir waves allowing the diffusion process to be supported until the wave energy is completely reabsorbed.

As seen from Eq. (1), all of the waves with phase velocities $u<v$ were involved in the acceleration of particles with a velocity $v$. Therefore, acceleration was basically provided by waves that were grown in a region with a positive growth rate. Wave propagation in inhomogeneous plasma suggests that part of the time waves pass in the region where their phase velocity is larger than the phase velocity of primar- ily generated waves, resulting in an interaction with particles having velocities higher than the velocity of particles of the beam that transfers part of their energy to waves.

Such an action allows the transfer of part of the wave energy to energetic particles, forming the tail of the distribution with energies larger than the beam energy. We used this process to describe the diffusion process toward higher energies. From Fig. 4 one can see that the population of fast electrons within the tail of the distribution function growth during beam relaxation (the filled areas). Therefore, electrons could be efficiently accelerated. Calculations with different initial conditions indicated that the energy of energetic particles depend on the initial $\Delta v$.

For $\Delta v<0.1 v_{0}$, more than $50 \%$ of the initial $\epsilon_{\mathrm{k}}$ could be transferred to a high energy tail due to the wave energy absorption by particles having energies higher than the energy of the beam. 


\section{Conclusions}

Electron beam relaxation in a plasma with a strongly inhomogeneous density fluctuation was numerically studied by making use of the system of equations derived by Ryutov (1969) in a quasi-linear approximation. The system describes beam-plasma interactions when the overwhelming majority of waves are trapped by density depletions. Under these conditions the growth rate is averaged along the wave path in the vicinity of the bottom of density wells. Two important features were observed during the relaxation of the beam that were different from the beam-plasma interaction in a homogeneous plasma. The relaxation process stops prior to the formation of a plateau-like distribution function because wave growth stops even when the slope of the distribution function remains positive locally. The relaxation process causes a transfer of a portion of the energy of waves to the high energy part of the electron velocity distribution. For the narrow beam $\left(\Delta v<0.1 v_{0}\right)$ the energy flux of accelerated electrons can reach up to $50 \%$ of the total energy flux of the beam. However, the presence of wave damping by the background distribution in the solar wind also impacts available wave energy that can be reabsorbed by the electron beam.

\section{Appendix A}

For the problem under consideration we assumed that the initial velocity of the beam, $v_{0}$, significantly exceeded the plasma thermal velocity, $v_{\mathrm{t}}$. The second assumption is that the initial width (variance) of the velocity, $\Delta v$, of the beam is small as compared to the beam velocity, $v_{0}$. We considered a beam moving in the direction of the magnetic field, parallel to the $x$ axis. Thus, our problem is 1-D. The density of a plasma is supposed to depend on the coordinates as follows: $n(x)=n_{0}+\Delta n(x)$, where $n_{0}$ is the unperturbed averaged value of the electron density and delta $\Delta n(x)$ describes small random deviations from $n_{0}$. An important additional condition is that the spatial scale of inhomogeneities, $a$, is supposed to be much larger than the wavelength of the Langmuir waves generated by the beam:

$a \omega_{\mathrm{p}} / v_{0} \gg 1$,

where $\omega_{\mathrm{p}}=\sqrt{4 \pi e^{2} n_{0} / m_{\mathrm{e}}}$ is the plasma frequency.

When inequality (A1) is satisfied, Langmuir waves in plasmas can be described as a superposition of the quasi-particles (wave packets) that corresponds to WKB description. The motion of these quasi-particles in the phase space can be described by the following Hamiltonian equations:

$\frac{d x}{d t}=\frac{\partial}{\partial k} \omega(\boldsymbol{k}, x)$

$\frac{d k}{d t}=-\frac{\partial}{\partial x} \omega(k, x)$ where $x$ is a coordinate, $\boldsymbol{k}$ is a momentum (wave vector), $\omega$ is the Hamiltonian function (frequency) that is the solution of the dispersion equation:

$\omega(\boldsymbol{k}, x)=\omega_{\mathrm{p}}\left(1+\frac{1}{2} \frac{n(x)}{n_{0}}\right)+\frac{3}{2} \frac{k^{2} v_{\mathrm{t}}^{2}}{\omega_{\mathrm{p}}}$.

Another important characteristic is the phase velocity, $v_{\mathrm{ph}}=$ $\omega / \boldsymbol{k}$. The interaction between an electron and a quasi-particle is possible only when $v=\omega / \boldsymbol{k}$.

The evolution of Langmuir wave spectral power can be described by the Liouville equation, as follows:

$\frac{\partial W}{\partial t}+\frac{\partial \omega}{\partial k} \frac{\partial W}{\partial x}-\frac{\partial \omega}{\partial x} \frac{\partial W}{\partial k}=2 \gamma W$,

where $\gamma$ is the growth rate of the beam instability, as follows:

$\gamma=\gamma(k, t)=\frac{\pi}{2} \frac{n^{\prime}}{n} \omega_{\mathrm{p}}\left(\frac{\partial f}{\partial v} v^{2}\right)_{v=v_{\mathrm{ph}}}$.

The presence of the density of the beam $n^{\prime}$ is due to the electron distribution function $f$, normalized to 1 as follows:

$\int_{0}^{\infty} f d v=1$.

The energy of the wave changes slowly on the time scale $a /\left(\frac{\partial \omega}{\partial k}\right)$ that allows one to describe the evolution of the wave spectral power, making use of the averaged growth rate. Using the variable $\omega$ instead of $k$ in Eq. (A5), as follows, is convenient:

$\frac{\partial W}{\partial t}+\frac{\partial \omega}{\partial k} \frac{\partial W}{\partial x}=2 \gamma W$.

In order to determine the solution to the equations above, we used the perturbation theory $W=W_{0}+\gamma W_{1}$ and took into account the fact that by using $\frac{\partial W_{0}}{\partial t} \sim 2 \gamma W_{0}$ one can obtain, in the first order approximation, the following:

$\frac{\partial W_{1}}{\partial x}=\frac{2 \gamma W_{0}-\partial W_{0} / \partial t}{\partial \omega / \partial k}$.

Let us consider quasi-particles that oscillate near the bottom of density wells. Then the following equation can be written

$\oint \frac{2 \gamma W_{0}-\partial W_{0} / \partial t}{\partial \omega / \partial k} d x=0$.

We integrate over the closed trajectories of quasi-particles. By considering that the $W_{0}$ does not depend on $x$ one can rewrite Eq. (A10), as follows:

$\frac{\partial W_{0}}{\partial t}=2 \bar{\gamma} W_{0}$, 
where $\bar{\gamma}$ is the averaged growth rate, as follows:

$\bar{\gamma}=\left(\oint \frac{\gamma}{\frac{\partial \omega}{\partial k}} d x\right) /\left(\oint \frac{d x}{\frac{\partial \omega}{\partial k}}\right)$,

In the vicinity of the bottom of the potential well, $x_{0}$, the density can be presented as follows:

$\Delta n(x)=\Delta n_{0}+\frac{1}{2}|\Delta n| \frac{\left(x-x_{0}\right)^{2}}{a_{0}^{2}}$,

where $\Delta n_{0}=\Delta n\left(x_{0}\right)$ and

$\frac{1}{a_{0}^{2}}=\frac{1}{\left|\Delta n_{0}\right|} \frac{\partial^{2} \Delta n}{\partial x^{2}}$.

Then, the Hamiltonian function reads as follows:

$\omega=$ const $+\omega_{\mathrm{p}} \frac{|\Delta n|}{2 n_{0}} \frac{\left(x-x_{0}\right)^{2}}{a_{0}^{2}}+\frac{3}{2} \frac{k^{2} v_{\mathrm{t}}^{2}}{\omega_{\mathrm{p}}}$.

Using Eq. (A15) one can integrate $\bar{\gamma}$ from Eq. (A12). Therefore, it is convenient to use the new variable of integration $v=\omega_{\mathrm{p}} / k(x)$, where $k(x)$ can be defined from the condition $\omega(x, k)=$ const. Easy to determine is that, as follows:

$\left(x-x_{0}\right)^{2}=\frac{a_{0}^{2} v_{\mathrm{t}}^{2}}{\varepsilon}\left(\frac{1}{u^{2}}-\frac{1}{v^{2}}\right), \quad \frac{\partial \omega}{\partial k}=3 \frac{v_{t}^{2}}{v}$,

where

$\varepsilon=\frac{\left|\Delta n_{0}\right|}{6 n_{0}}, \quad u=\frac{\omega_{\mathrm{p}}}{k\left(x_{0}\right)}$,

where $u$ is the phase velocity of the quasi-particle in the bottom of the well. Using Eqs. (A16), (A12) and (A6) one can obtain the following:

$\bar{\gamma}(u, t)=\frac{\omega_{\mathrm{p}} n^{\prime}}{2 n} \int_{u}^{\infty} \frac{\partial f(v, t)}{\partial v}\left(\frac{1}{u^{2}}-\frac{1}{v^{2}}\right)^{-\frac{1}{2}} d v$.

The factor $\sim \pi$ from Eq. (A6) is canceled as a result of the spatial averaging in Eq. (A12).

Now we consider the following equation for the electron distribution function:

$\frac{\partial f}{\partial t}+v \frac{\partial f}{\partial x}=\frac{\partial}{\partial v} D \frac{\partial f}{\partial v}$,

where $D$ is the quasi-linear diffusion coefficient, as follows:

$D=\frac{4 \pi^{2} e^{2}}{m^{2} v} W(k, x, t)$.

By taking into account the inequality (A1) and by assuming that the oscillations in each single cavity are independent on those in other cavities, one can average over $x$ to obtain the following:

$\frac{\partial \bar{f}}{\partial t}=\frac{\partial}{\partial v} \bar{D} \frac{\partial \bar{f}}{\partial v}$ where $\bar{D}$ is the averaged diffusion coefficient and $\bar{f}$ is the averaged electron distribution function, as follows:

$\bar{D}=\lim _{L \rightarrow \infty} \frac{1}{2 L} \int_{-L}^{L} D(x, k, t) d x$.

For the quasi-particles that oscillate in the vicinity of the bottom of the potential well from the Eqs. (A16), (A22) one can find the diffusion coefficient, as follows:

$\bar{D}=\frac{8 \pi^{2} e^{2}}{m^{2}} v_{\mathrm{t}} \int_{0}^{v} \frac{\bar{W}(u, t)}{u^{2} \sqrt{v^{2}-u^{2}}} d u$,

where

$\bar{W}(u, t)=\lim _{L \rightarrow \infty} \frac{1}{2 L} \frac{a_{0} W_{0}}{\sqrt{\varepsilon}}$.

The complete system of equations describing the relaxation of the electron beam in inhomogeneous plasmas in the quasilinear approximation is, as follows:

$\frac{\partial \bar{f}}{\partial t}=\frac{\partial}{\partial v} \bar{D} \frac{\partial \bar{f}}{\partial v}, \bar{D}=\frac{8 \pi^{2} e^{2}}{m^{2}} v_{\mathrm{t}} \int_{0}^{v} \frac{\bar{W}}{u^{2} \sqrt{v^{2}-u^{2}}} d u$,

$\frac{\partial \bar{W}}{\partial t}=2 \bar{\gamma} \bar{W}, \bar{\gamma}=\frac{1}{2} \omega_{\mathrm{p}} \frac{n^{\prime}}{n} u \int_{u}^{\infty} \frac{v \partial \bar{f} / \partial v}{\sqrt{v^{2}-u^{2}}} d v$.

After a substitution of the variables $\bar{f} \rightarrow \frac{\bar{f}}{v_{0}}, \bar{W} \rightarrow \frac{n^{\prime} m v_{0}^{4}}{\pi \omega_{\mathrm{p}} v_{t}} \bar{W}$, $t \rightarrow \frac{n}{n^{\prime} \omega_{\mathrm{p}}} t, v=v_{0} v, u=v_{0} u$, one can obtain the following dimensionless system:

$\frac{\partial \bar{f}}{\partial t}=\frac{\partial}{\partial v}\left(\int_{0}^{v} \frac{\bar{W}}{u^{2} \sqrt{v^{2}-u^{2}}} d u\right) \frac{\partial \bar{f}}{\partial v}$

$\frac{\partial \bar{W}}{\partial t}=\bar{W} u \int_{u}^{\infty} \frac{v \frac{\partial \bar{f}}{\partial v}}{\sqrt{v^{2}-u^{2}}} d v$

For this work, we solve this system (A27, A28) numerically aiming to study the process of quasi-linear relaxation of the electron beam in inhomogeneous plasmas.

Acknowledgements. We are grateful to A. Artemyev and O. Agapitov for helpful discussions. The work of V. Krasnoselskikh was supported by a grant from CNES - "Invited scientist in Stereo Waves experiment".

Guest Editor M. Balikhin thanks P. J. Kellogg and one anonymous referee for their help in evaluating this paper. 


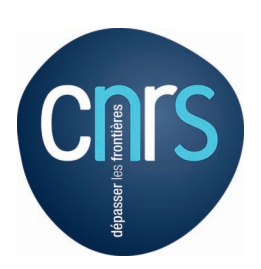

The publication of this article is financed by CNRS-INSU.

\section{References}

Buttighoffer, A.: Solar electron beams associated with radio type III, A\&A., 335, 295-302, 1998.

Celnikier, L. M., Harvey, C. C., Jegou, R., Moricet, P., and Kemp, M.: A determination of the electron density fluctuation spectrum in the solar wind, using the ISEE propagation experiment, A\&A., 126, 293-298, 1983.

Drummond, W. E. and Pines, D.: NON-LINEAR STABILITY OF PLASMA OSCILLATIONS, Nuclear Fusion, 2, 1049-1057, 1962.

Ergun, R. E., Malaspina, D. M., Cairns, I. H., Goldman, M. V., Newman, D. L., Robinson, P. A., Eriksson, S., Bougeret, J. L., Briand, C., Bale, S. D., Cattell, C. A., Kellogg, P. J., and Kaiser, M. L.: Eigenmode Structure in Solar-Wind Langmuir Waves, Phys. Rev. Lett., 101, 051101, doi:10.1103/PhysRevLett.101.051101, 2008.

Foroutan, G., Khalilpour, H., Moslehi-Fard, M., Li, B., and Robinson, P. A.: Quasilinear dynamics of a cloud of hot electrons propagating through a plasma with decreasing density and temperature, Phys. Plasmas, 15, 122904, doi:10.1063/1.3046179, 2008.

Ginzburg, V. L. and Zhelezniakov, V. V.: On the Possible Mechanisms of Sporadic Solar Radio Emission (Radiation in an Isotropic Plasma), Soviet. Ast., 2, 653-678, 1958.

Grognard, R. J.-M.: Numerical simulation of the weak turbulence excited by a beam of electrons in the interplanetary plasma, Solar Phys., 81, 173-180, 1982.

Kellogg, P. J.: Observations concerning the generation and propagation of Type III solar bursts, A\&A., 169, 329-335, 1986.

Kellogg, P. J., Goetz, K., Monson, S. J., and Bale, S. D.: Langmuir waves in a fluctuating solar wind, J. Geophys. Res., 104, 1706917078, 1999.

Kontar, E. P. and Reid, H. A. S.: Onsets and Spectra of Impulsive Solar Energetic Electron Events Observed Near the Earth, ApJ Lett., 695, L140-L144, 2009.

Li, B., Robinson, P. A., and Cairns, I. H.: Quasilinear calculation of Langmuir wave generation and beam propagation in the presence of density fluctuations, Phys. Plasmas, 13, 082305, doi:10.1063/1.2218331, 2006.
Lin, R. P.: The Emission and Propagation of $40 \mathrm{keV}$ Solar Flare Electrons. I: The Relationship of $40 \mathrm{keV}$ Electron to Energetic Proton and Relativistic Electron Emission by the Sun, Solar Phys., 12, 266-303, 1970.

Lin, R. P., Potter, D. W., Gurnett, D. A., and Scarf, F. L.: Energetic electrons and plasma waves associated with a solar type III radio burst, ApJ, 251, 364-373, 1981.

Papadopoulos, K., Goldstein, M. L., and Smith, R. A.: Stabilization of Electron Streams in Type III Solar Radio Bursts, ApJ, 190, 175-186, 1974.

Parker, E. N.: Interplanetary Origin of Energetic Particles, Phys. Rev. Lett., 14, 55-57, doi:10.1103/PhysRevLett.14.55, 1965.

Reid, H. A. S. and Kontar, E. P.: Solar Wind Density Turbulence and Solar Flare Electron Transport from the Sun to the Earth, ApJ, 721, 864-874, 2010.

Ryutov, D. D.: Quasilinear Relaxation of an Electron Beam in an Inhomogeneous Plasma, Soviet Journal of Experimental and Theoretical Physics, 30, 131-137, 1969.

Samarskii, A. A. and Popov, I. P.: Finite-difference schemes of gasdynamics, Moscow Nauka, 2, 50-87, 1975.

Shapiro, V. D. and Shevchenko, V. I.: Strong turbulence of plasma oscillations, in: Basic Plasma Physics: Selected Chapters, Handbook of Plasma Physics, Volume 1, edited by: Galeev, A. A. and Sudan, R. N., p. 123, 1984.

Sturrock, P. A.: Type III Solar Radio Bursts, NASA Special Publication, 50, 357-364, 1964.

Takakura, T. and Shibahashi, H.: Dynamics of a cloud of fast electrons travelling through the plasma, Solar Phys., 46, 323-346, 1976.

Vedenov, A. A., Velikhov, E., and Sagdeev, R.: Quasilinear theory of plasma oscillations, Nuclear Fusion Suppl., 2, 465-475, 1962.

Zaitsev, V. V., Mityakov, N. A., and Rapoport, V. O.: A Dynamic Theory of Type III Solar Radio Bursts, Solar Phys., 24, 444-456, 1972.

Zakharov, V. E.: Collapse of Langmuir Waves, Soviet Journal of Experimental and Theoretical Physics, 35, 908-914, 1972.

Zaslavsky, A., Volokitin, A. S., Krasnoselskikh, V. V., Maksimovic, M., and Bale, S. D.: Spatial localization of Langmuir waves generated from an electron beam propagating in an inhomogeneous plasma: Applications to the solar wind, J. Geophys. Res. (Space Physics), 115, A08103, doi:10.1029/2009JA014996, 2010.

Ziebell, L. F., Yoon, P. H., Pavan, J., and Gaelzer, R.: Nonlinear Evolution of Beam-plasma Instability in Inhomogeneous Medium, ApJ, 727, 16-26, 2011. 